\title{
"Michael" Nanocarriers Mimicking Transient-Binding Disordered Proteins
}

\author{
Ana Sanchez-Sanchez, ${ }^{1}$ Somayeh Akbari, ${ }^{2}$ Agustín Etxeberria, ${ }^{3}$ Arantxa Arbe, ${ }^{1}$ Urs Gasser, ${ }^{4}$ Angel J. \\ Moreno, ${ }^{1}$ Juan Colmenero, ${ }^{1,5,6}$ and José A. Pomposo ${ }^{*}, 1,5,7$ \\ ${ }^{1}$ Centro de Física de Materiales (CSIC, UPV/EHU)-Materials Physics Center, Paseo Manuel de Lardizabal 5, 20018 San Sebastián, Spain \\ ${ }^{2}$ Laboratory for Tribology and Surface Nanotechnology, Bogišičeva 8, 1000 Ljubljana, Slovenia \\ ${ }^{3}$ Departamento de Ciencia y Tecnología de Polímeros, Universidad del País Vasco (UPV/EHU), Paseo Manuel de Lardizabal 3, 20018 San \\ Sebastián, Spain \\ ${ }^{4}$ Laboratory for Neutron Scattering, Paul Scherrer Institut, CH-5232 Villigen, Switzerland \\ ${ }^{5}$ Departamento de Física de Materiales, Universidad del País Vasco (UPV/EHU), Apartado 1072, 20800 San Sebastián, Spain \\ ${ }^{6}$ Donostia International Physics Center (DIPC), Paseo Manuel de Lardizabal 4, 20018 San Sebastián, Spain \\ ${ }^{7}$ IKERBASQUE - Basque Foundation for Science, Alameda Urquijo 36, 48011 Bilbao, Spain
}

\section{Supporting Information}

\begin{abstract}
We report herein a very efficient synthesis strategy for the construction of artificial transient-binding protein-mimic nano-objects. Michael addition-mediated multidirectional self-assembly of individual polymeric chains at r.t. leads to "Michael" nanocarriers that in solution resemble disordered multidomain proteins, as revealed by a combination of small angle neutron scattering (SANS) measurements and coarse-grained molecular dynamics (MD) results, whereas in the dry state adopt a collapsed, globular morphology, as observed by transmission electron microscopy (TEM). This extended-to-compact morphology transition taking place upon solvent removal is of paramount importance, among other applications, for the construction of efficient biosensors based on immobilized protein-mimic nano-objects and for the development of transient vitamin-binding systems. As a proof of concept, we show the controlled delivery of vitamin $\mathrm{B}_{9}$ from these novel transient-binding nanocarriers.
\end{abstract}

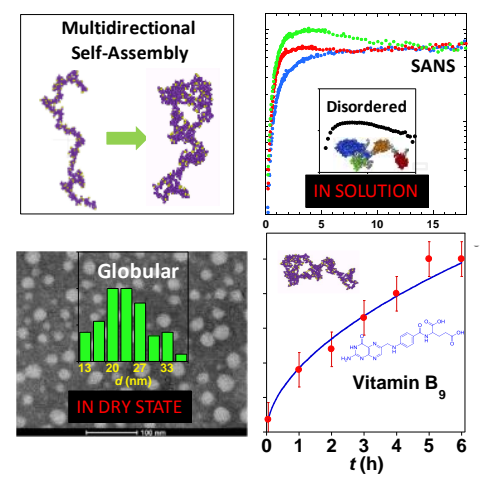

Nature is a continuous source of inspiration for scientists across different disciplines. ${ }^{1}$ In particular, the specific - native- conformation of proteins allowing these large biomolecules to carry out sophisticated tasks such as catalysis inspired the construction of a first generation of artificial enzymes based on a variety of molecular and macromolecular structures such as macrocyclic compounds, ${ }^{2}$ star and helical polymers, ${ }^{3}$ dendrimers ${ }^{4}$ and micelles. ${ }^{5}$ Single-chain polymer nanoparticles are emerging soft nanoobjects showing unique and remarkable physicochemical, rheological and sensing properties, ${ }^{6}$ as a result of their locally collapsed structure and its ultra-small size. $\mathrm{We}^{7}$ and others ${ }^{8,9}$ have demonstrated that catalytic properties can be imparted to singlechain nanoparticles through polymer folding/collapse accompanied by efficient catalyst immobilization. The potential use of single-chain nanoparticles as drug / siRNA nanocarriers and photostable bioimaging agents relies on the improved construction of functional folded/collapsed single polymer chains. Moreover, controlled synthesis of single-chain nanocarriers becomes critical to the elucidation of useful structure-property relationships in materials science and to deepen our current understanding of complex cooperative folding events taking place in synthetic single polymer chains emulating protein folding. ${ }^{10}$ Unfortunately, most of the current synthesis routes to stable single-chain nanoparticles suffer from different shortcomings, such as use of extremely high temperatures, requirement of severe anhydrous conditions, involvement of metallic catalysts or necessity of exotic, non-commercial monomers, which severely limit their potential applications in some promising fields (e.g., nanomedicine). ${ }^{11}$ Despite all recent advances, the development of a natural route to well-defined single-chain nano-objects allowing to investigate how far, or close, are the structure and function of these synthetic nanocarriers from those of globular, or intrinsically disordered, proteins is still a challenging issue.

In this letter, we report a simple and highly-efficient bioinspired method for transient-binding nanocarrier construction that relies on multidirectional self-assembly of individual polymeric chains at r.t. driven by multiple intrachain Michael addition reactions involving external multifunctional acrylate-based crosslinkers (see Scheme 1). Single-chain nano-object formation through multidirectional self-assembly is inspired by protein assembly to the folded state. For proteins, the driving force for folding depends on the sequence of amino acids, their mutual interactions and their interactions with solvent molecules, whereas for "Michael" nanocarriers the driving force is replaced by multiple, cooperative, omnidirectional chemical (Michael addition) reactions taking place across the collapsing polymer chains under good solvent conditions. The Michael reaction has been previously employed in the synthesis of linear, graft, hyperbranched, den- 


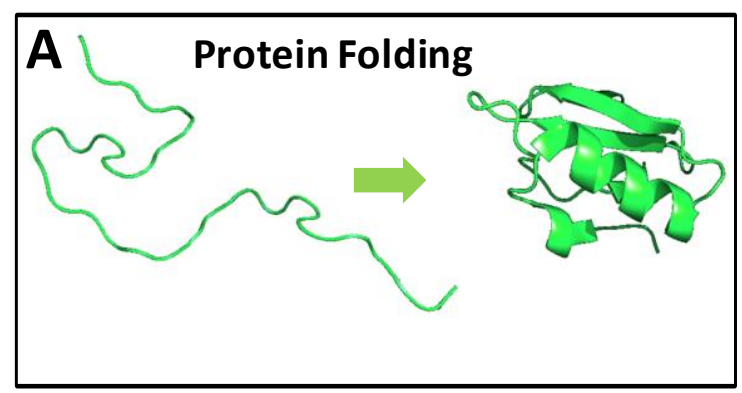

B Multidirectional Self-Assembly
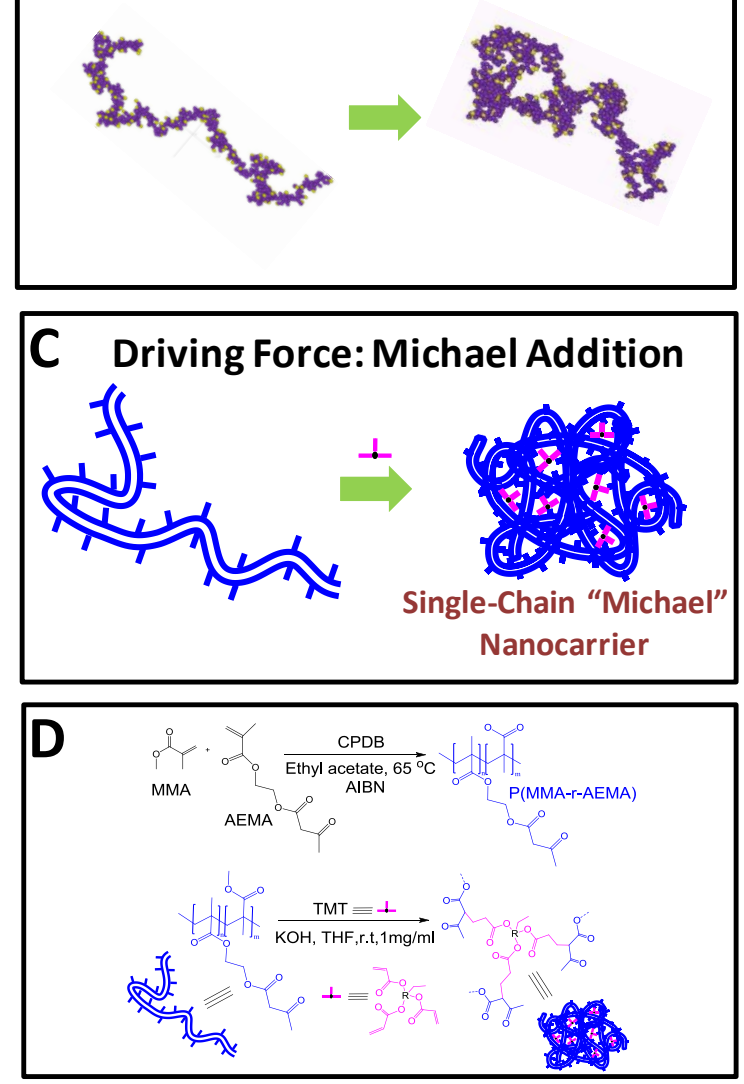

Scheme 1. Analogy between protein folding (A) and multidirectional self-assembly during the synthesis of "Michael" nanocarriers (B). The driving force for nanocarrier formation in B is intramolecular Michael addition reaction taking place in a multiple, cooperative and omnidirectional manner at room temperature $(\mathrm{C})$ involving external multifunctional acrylate-based cross-linkers that react with $\beta$-ketoester groups of the unfolded polymer chain under appropriate solvent, stoichiometric and dilution conditions (D).

dritic and network polymers, as well as in post-polymerization modification and bioconjugation, being one of the most useful and versatile reactions to create new carbon-carbon bonds. ${ }^{12}$

As shown below in this communication, the benefits of Michael addition-mediated multidirectional self-assembly as a synthesis strategy for artificial protein-mimic nano-objects are: $i$ ) versatility of the method in terms of reaction conditions (i.e., absence of metal catalysts, room temperature, air atmosphere), ii) commercial availability of reagents allowing the facile synthesis of Michael nanocarriers (gram-scale) for potential end-use in nanomedicine, bioimaging and sensing applications, iii) convenient time-span for direct monitoring in solution of the self-organized, cooperative folding/collapse process, $i v$ ) versatility for obtaining nano-objects resembling in solution transient-binding disordered proteins, and v) promising nanocarrier properties of the resulting multidomain nano-objects.

"Michael" nanocarriers were prepared from polymeric precursors synthesized through reversible addition-fragmentation chaintransfer (RAFT) polymerization by starting with commercially available methyl methacrylate (MMA) and (2-acetoacetoxy)ethyl methacrylate (AEMA) monomers (SI, Schemes S1-S2). Statistical copolymers were obtained due to the similar reactivity ratios of MMA $(r=0.90)$ and AEMA $(r=0.95)$ providing materials of high molar mass, relatively narrow size dispersity and random distribution of $\beta$-ketoester functional groups (i.e., Michael donors) along the polymer chain. As external cross-linkers playing the role of Michael acceptors, we selected commercially available low-molecular-weight multi-functional (bi-, tri-, tetra-, penta / hexa-) acrylate compounds (see SI, Scheme S1). The multidirectional self-assembly of the individual polymeric precursor to Michael nanocarriers was performed in tetrahydrofuran (THF), at r.t. under potassium hydroxide catalysis and air atmosphere, at a concentration of polymeric precursor of $1 \mathrm{mg} / \mathrm{ml}$ by using equimolar amounts of $\beta$-ketoester and acrylate functional groups. As an example, Figure 1A shows the systematic shift in size exclusion chromatography (SEC) traces towards longer retention time during the synthesis of single-chain Michael nanocarriers in the presence of a tri-functional cross-linker (trimethylolpropane triacrylate, TMT). The noticeable shift observed is a consequence of the progressive reduction in hydrodynamic size and, consequently, in apparent molar mass $\left(M_{p}^{a p p}\right)$ with reaction time (open circles in Figure 1A). ${ }^{13}$ The absence of significant inter-particle aggregation was confirmed through simultaneous static light scattering (SLS) measurements from which, as expected, a nearly constant value of actual molar mass was observed $\left(M_{p} \approx 293 \mathrm{kDa}\right.$, closed circles in Figure 1A, Figures S1-S3). ${ }^{14,15}$ Under identical reaction conditions (i.e., solvent, temperature, reagent concentration) the kinetics of the Michael addition reaction for multidirectional self-assembly was $c a$. 50-fold slower than that corresponding to condensation via Michael addition of mixtures of lowmolecular-weight model compounds (e.g., methyl acetoacetate / TMT mixtures) as determined by ${ }^{1} \mathrm{H}$ NMR measurements (Figure 1B, Figures S4-S5). The main reason for the significantly slower kinetics seems to be the macromolecular character of the polymeric precursor which imposes diffusional constrains and significantly restricted intrachain accessibility to the low-molecular-weight acrylate cross-linker units.

We have determined the kinetics of the folding/collapse process in solution through the direct monitoring of the value of z-average radius of gyration $\left(R_{g}\right)$ of the polymeric precursor as a function of reaction time, by means of static light scattering (SLS) measurements (Figure 1C). The kinetics of the folding/collapse process was found to be $c a$. 5-fold slower than that of the Michaelmediated intrachain cross-linking reaction, which can be attributed to the coexistence of non-efficient and efficient folding events. In this sense, the first anchoring step of a multifunctional crosslinker molecule through reaction of a single acrylate group can be considered as a non-efficient folding event that does not contribute to chain collapse. Conversely, the delayed, subsequent intrachain Michael reactions of the remaining acrylate groups are truly folding/collapse-promoting events. As illustrated in Figure 1C, the kinetics of folding/collapse is slightly faster upon decreasing the molar mass of the polymeric precursor. Even displaying much slower folding kinetics when compared to that of natural proteins (from microseconds to hours), ${ }^{16}$ the resulting Michael nanocarrriers in the dried state showed a compact spherical morphology as illustrated in Figure 1D. 

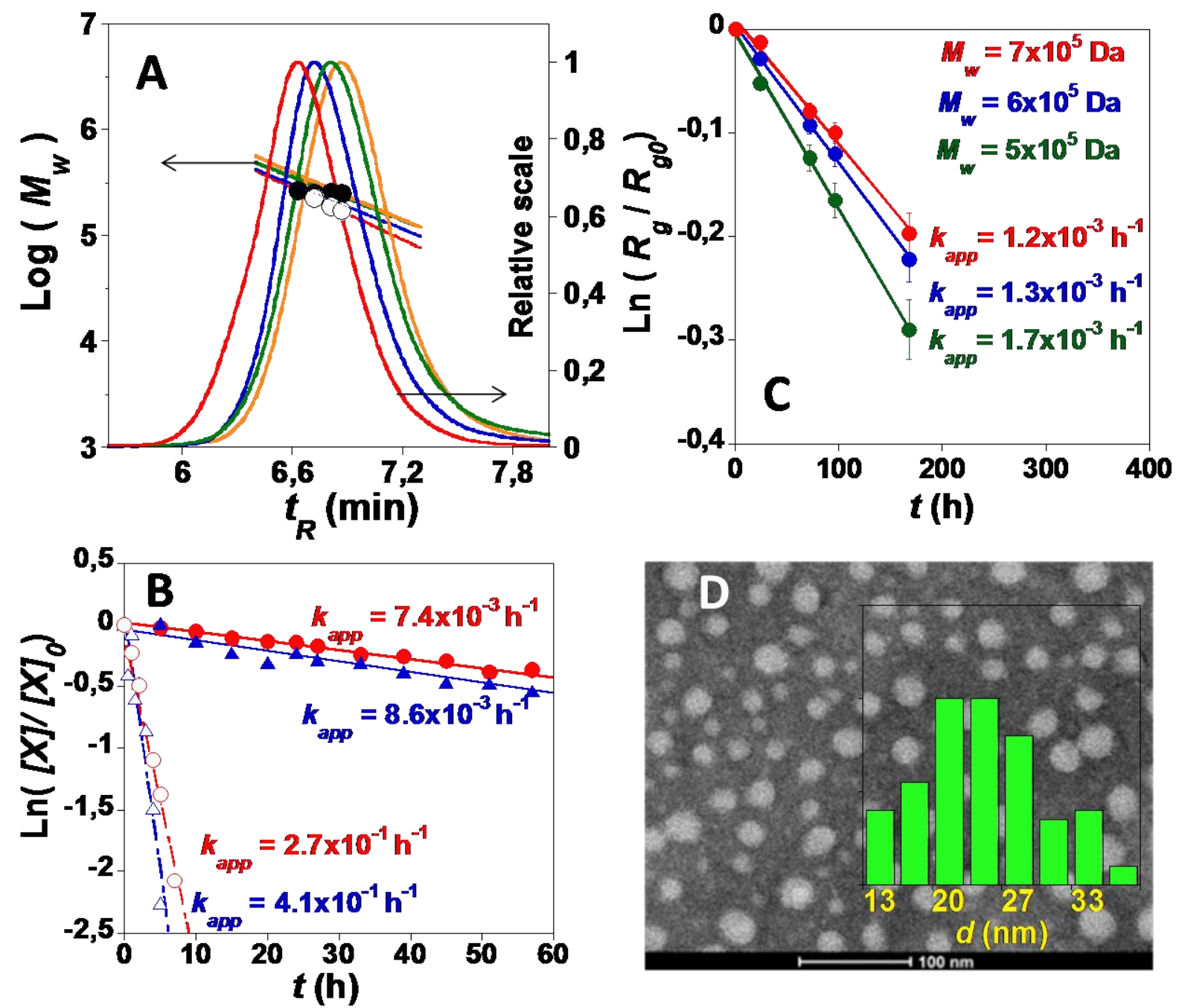

Figure 1. "Michael" nanocarrier construction through multidirectional self-assembly: (A) The shift in SEC traces upon reaction time (from left to right: $0 \mathrm{~h}, 17 \mathrm{~h}, 48 \mathrm{~h}$ and $72 \mathrm{~h})$ is due to a progressive reduction in hydrodynamic volume and, consequently, in apparent molar mass, $\left(\mathrm{M}_{\mathrm{p}}{ }^{\mathrm{app}}\right.$, open symbols), whereas the nearly constant value of actual molar mass $\left(\mathrm{M}_{\mathrm{p}} \approx 293 \mathrm{kDa}\right.$, closed symbols) points to the collapse of individual polymer chains without significant inter-particle aggregation. (B) Pseudo-first order kinetics plots corresponding to the consumption of bi-functional (closed circles) and tri-functional (closed triangles) cross-linker units (X). Also displayed for comparison are the corresponding kinetics plots for low-molecular-weight model compound mixtures (open symbols). (C) Pseudo-first order kinetics plots corresponding to the folding/collapse process in solution at r.t. in the presence of tri-functional cross-linker units. (D) TEM image showing the morphology of Michael nanocarriers in the dry state.

Upon isolation by precipitation, drying under dynamic vacuum and further solubilization, Michael nanocarriers were found to form transparent, colorless dispersions in common organic solvents (tetrahydrofuran, chloroform, dimethyl formamide). Unfortunately, all our attempts to synthesize well-defined Michael nanocarriers by means of tetra- or penta/hexa-acrylate cross-linker molecules failed suggesting that under our reaction conditions the optimum cross-linker functionality is $f=3$. We suspect that the increased steric hindrance for cross-linkers of $\mathrm{f}>3$ and the higher tendency to form insoluble macroscopic gels due to inter-particle coupling events could be responsible of this behavior.

Valuable information about the actual structure in solution of single-chain Michael nanocarriers synthesized by means of biand tri-functional cross-linker units was obtained through small angle neutron scattering (SANS) measurements in deuterated solvent and complementary molecular dynamics (MD) simulations. Figure 2A shows Kratky plots for the unfolded polymer chains and the Michael nanocarriers synthesized in the presence of bi- and tri-functional cross-linkers. It is worth mentioning that multidirectional self-assembled Michael nanocarriers ( $f=3$ ) display a clear maximum in the Kratky plot in resemblance to partially folded proteins. For globular proteins, it is well-known that the significant decrease observed in $\mathrm{IQ}^{2}(\mathrm{I}=$ scattered intensity, $Q=$ scattering vector) at large $Q$ is characteristic of a compact structure. ${ }^{17}$ However, Kratky plots of Michael nanocarriers in solution more closely resemble those of disordered multidomain proteins with semiflexible linkers (e.g., p6 $\left.{ }^{\text {phox }}\right)^{18,19}$ than those expected for globular proteins (e.g., polX). ${ }^{20}$ Kratky plots from MD simulations results corresponding to the unfolded polymeric precursor and the resulting nano-objects are drawn in Figure 2B. It is worth noting the qualitative agreement between SANS and MD simulation results. For both kinds of nanoparticles, MD simulation data showed increased compact conformations on decreasing the quality of the solvent, which is consistent with the globular conformation observed by TEM for the Michael nanocarriers in the dry state (Figure 1D). 

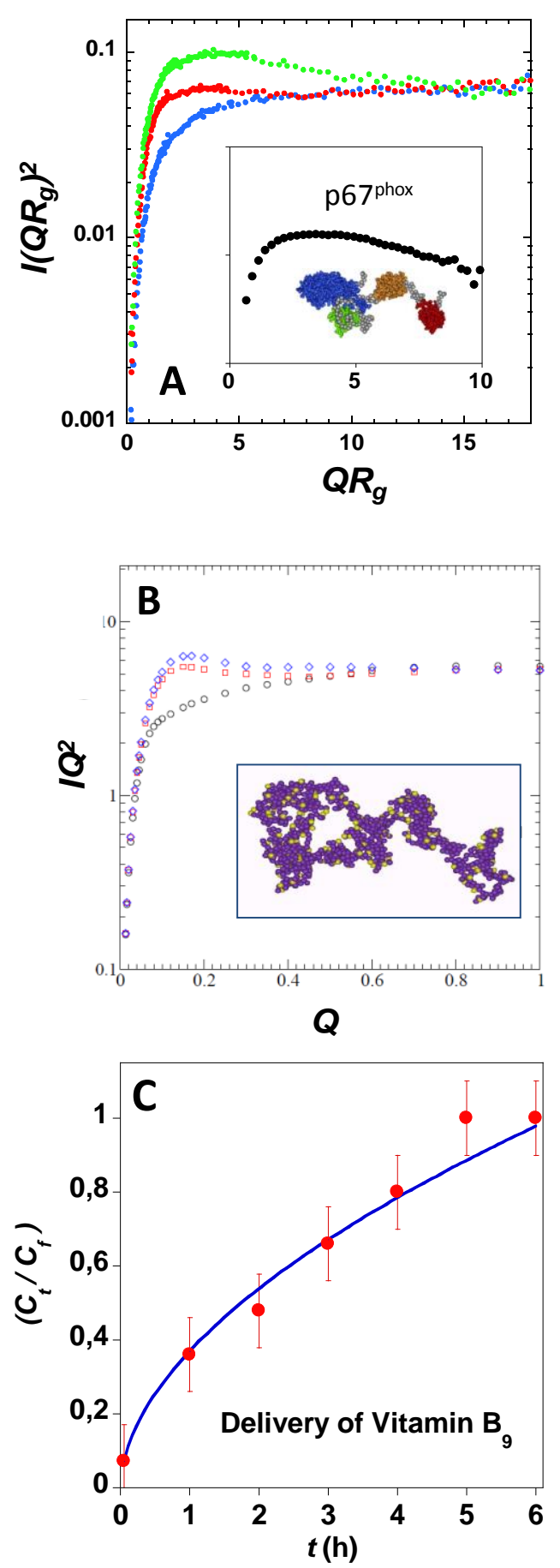

Figure 2. "Michael" nanocarriers in solution: (A) Kratky plots for solutions of unfolded polymer chains (blue symbols) and Michael nanocarriers synthesized with cross-linkers of functionality $f=2$ (red symbols) and $\mathrm{f}=3$ (green symbols), as determined by SANS. Note that multidirectionally self-assembled Michael nano-objects $(f=3)$ display a clear maximum in the Kratky plot in resemblance to disordered multi-domain proteins with semi-flexible linkers (see inset taken from ref. 18). (B) Kratky plots from MD simulations under good solvent conditions of linear precursor (black symbols) and Michael nano-objects of functionality $\mathrm{f}=2$ (red symbols) and $\mathrm{f}=3$ (blue symbols). The inset shows a typical snapshot from the MD simulations of the nano-object conformation. (C) Delivery curve in water at neutral $\mathrm{pH}$ from vitamin $\mathrm{B}_{9}$-loaded Michael nanocarriers (red symbols) and best-fit of the experimental data to the well-known power law model $\left(C_{t} / C_{f}=K t^{n}\right.$, see text) (blue line).
Inspired by the behavior of natural transient-binding disordered proteins, we have investigated the drug-delivery properties of Michael nanocarriers synthesized with tri-functional cross-linker units for potential application in dermal supply of vitamin $\mathrm{B}_{9}$ (i.e., folate or folic acid). Several in vitro and in vivo studies have indicated that this essential vitamin may offer a treatment option for photo-aged skin when incorporated in topical formulations. ${ }^{21}$ Hence, vitamin $\mathrm{B}_{9}$ nanocarriers based on single-chain Michael nanoparticles were placed in distilled water at neutral $\mathrm{pH}$ to investigate their controlled delivery properties. Figure $2 \mathrm{C}$ illustrates the progressive delivery of vitamin $\mathrm{B}_{9}$ from the single-chain Michael nanocarriers as determined from UV/Vis spectroscopy measurements at $283 \mathrm{~nm}$. The continuous line is a best-fit to the wellknown power law model $\left(C_{t} / C_{f}=K t^{n}, C_{t}=\right.$ concentration of drug released at time $t, C_{f}=$ total concentration of drug released, $K=$ constant, $n=$ release exponent). ${ }^{22}$ The value obtained for the release exponent, $n \approx 0.5$, suggests that the delivery process proceeds through a Fickian diffusion mechanism. Complete delivery of vitamin $B_{9}$ from Michael nanoparticles with a drug loading content of $41 \mathrm{wt} \%$ was observed to take place in 5-6 h. These promising results pave the way for mimicking the multiple transient-binding behavior of intrinsically disordered proteins (IDPs) ${ }^{23}$ using Michael nanocarriers.

In summary, we have developed a highly-efficient strategy for the permanent multidirectional self-assembly of unfolded polymeric chains to Michael nanocarriers that in solution resemble disordered multidomain proteins with semiflexible linkers. Conversely, in the dry state they adopt a collapsed, globular morphology. This natural synthesis route proceeds under very mild reaction conditions (without metal catalysts, at room temperature, under air atmosphere) by starting with commercially available reagents, allowing the efficient preparation of novel "Michael" nanocarriers showing multiple, locally compact domains in solution that turn to a collapsed, globular morphology in the dry state. By using single-chain Michael nano-objects as vitamin $B_{9}$ nanocarriers, sustained release of vitamin $B_{9}$ in water at neutral $\mathrm{pH}$ following a Fickian diffusion mechanism has been demonstrated. Both the multidirectional self-assembly approach, as a synthesis strategy to nano-objects resembling partially folded multidomain proteins in solution, and the own Michael nanocarriers, resembling transient-binding IDPs, are expected to find applicability in different fields, such as nanomedicine, biosensing / bioimaging uses, and heterogeneous catalysis.

\section{ASSOCIATED CONTENT}

Supporting Information. Materials, methods, characterization techniques, molecular dynamics (MD) simulations and supporting data. This material is available free of charge via the Internet at http://pubs.acs.org.

\section{AUTHOR INFORMATION}

\section{Corresponding Author}

* E-mail: Josetxo.pomposo@ehu.es

\section{Author Contributions}

The manuscript was written through contributions of all authors

Notes

The authors declare no competing financial interest.

\section{ACKNOWLEDGMENT}

Financial support from the projects MAT2012-31088 (MINECO) and IT-654-13 (GV) is acknowledged. A. S.-S. thanks the PhD grant support of Basque Government. This work is based on experiments performed at the Swiss spallation neutron source 
SINQ, Paul Scherrer Institute, Villigen, Switzerland and has been supported by the European Commission under the $7^{\text {th }}$ Framework Programme through the 'Research Infrastructures' action of the 'Capacities' Programme, NMI3-II Grant number 283883.

\section{REFERENCES}

(1) Studart, A. R. Adv. Mater. 2012, 24, 5024-5044.

(2) Breslow, R.; Dong, S. D. Chem. Rev. 1998, 98, 1997-2011.

(3) a) Inoue, K. Prog. Polym. Sci. 2000, 25, 453-571. b) Yamamoto,

T.; Yamada, T.; Nagata, Y.; Suginome, M. J. Am. Chem. Soc. 2010, 132, 7899-7901.

(4) Liang, C.; Fréchet, J. M. J. Prog. Polym. Sci. 2005, 30, 385.

(5) Wang, Y.; Xu, H.; Ma, N.; Wang, Z.; Zhang, X.; Liu, J.; Shen, J. Langmuir 2006, 22, 5552-5555.

(6) a) Mackay, M. E.; Dao, T. T.; Tuteja, A.; Ho, D. L.; Horn, B. V.; Kim, H.-C.; Hawker, C. J. Nat. Mater. 2003, 2, 762-766. b) Oria, L.; Aguado, R.; Pomposo, J. A.; Colmenero, J. Adv. Mater. 2010, 22, 3038-3041. c) Gillissen, M. A. J.; Voets, I. K.; Meijer, E. W.; Palmans, A. R. A. Polym. Chem. 2012, 3, 3166-3174.

(7) Pérez-Baena, I.; Barroso-Bujans, F.; Gasser, U.; Arbe, A.; Moreno, A. J.; Colmenero, J.; Pomposo, J. A. Chem. Sci. 2013 (submitted). (8) Wulff, G.; Chong, B.-O.; Kolb, U. Angew. Chem. Int. Ed. 2006, 45, 2955-2958

(9) Terashima, T.; Mes, T.; De Greef, T. F. A.; Gillissen, M. A. J.; Besenius, P.; Palmans, A. R. A.; Meijer, E. W. J. Am. Chem. Soc. 2011, 133, 4742-4745.

(10) Dill, K. A.; MacCallum, J. L. Science 2012, 338, 1042-1046.

(11) Altintas, O.; Barner-Kowollik, C. Macromol. Rapid Commun. 2012, 33. 958-971.

(12) Mather, B. D.; Viswanathan, K.; Miller, K. M.; Long, T. E. Prog. Polym. Sci. 2006, 31, 487-531.

(13) Pomposo, J. A.; Perez-Baena, I.; Buruaga, L.; Alegría, A.; Moreno, A. J.; Colmenero, J. Macromolecules 2011, 44, 8644-8649.

(14) Tuten, B. T.; Chao, D.; Lyon, C. K.; Berda, E. B. Polym. Chem. 2012, 3, 3068-3071.

(15) Chao, D.; Jia, X.; Tuten, B.; Wang, C.; Berda, E. B. Chem. Commun. 2013 (In press). DOI: 10.1039/C2CC37157J.

(16) Ivankov, D. N.; Finkelstein, A. V. PNAS 2004, 101, 8942-8944.

(17) Pollack, L.; Tate, M. W.; Darnton, N. C.; Knight, J. B.; Gruner, S. M.; Eaton, W. A.; Austin, R. H. PNAS 1999, 96, 10115-10117.

(18) Durand, D.; Vives, C.; Cannella, D.; Perez, J.; Pebay-Peyroula, E.; Vachette, P.; Fieschi, F.; J. Struct. Biol. 2010, 169, 45-53.

(19) We are not claiming that "Michael" nanocarriers have similarity to the precise sequence of proteins but that their form factor in solution resembles that of disordered proteins, as a consequence of the multidirectional self-assembly process which leads to the formation of local globules along the individual chains. In resemblance to transient-binding disordered proteins, the resulting Michael nanocarriers are able to bind temporally vitamin $\mathrm{B}_{9}$ that can be delivered in a controlled manner (Figure 2C).

(20) Receveur-Brechot, V.; Durand, D. Curr. Protein Pept. Sc. 2012, $13,55-75$.

(21) a) Knott, A.; Mielke, H.; Koop, U.; Wolber, R.; Burkhardt, T.; Vietzke, J.-P.; Stäb, F.; Wenck, H.; Gallinat, S. J. Invest. Dermatol. 2007, 127, 2463-2466. b) Fischer, F.; Achterberg, V.; März, A.; Puschmann, S.; Rahn, C.-D.; Lutz, V.; Krüger, A.; Schwengler, H.; Jaspers, S.; Koop, U.; Blatt, T.; Wenck, H.; Gallinat, S. J. Cosmet. Dermatol. 2011, 10, 15-23.

(22) Laoa, L. L.; Peppas, N. A.; Boeya, F. Y. C.; Venkatramana, S. S. Int. J. Pharm. 2011, 418, 28-41.

(23) Tompa, P. FEBS Lett. 2005, 579, 3346-3354. 
Insert Table of Contents artwork here
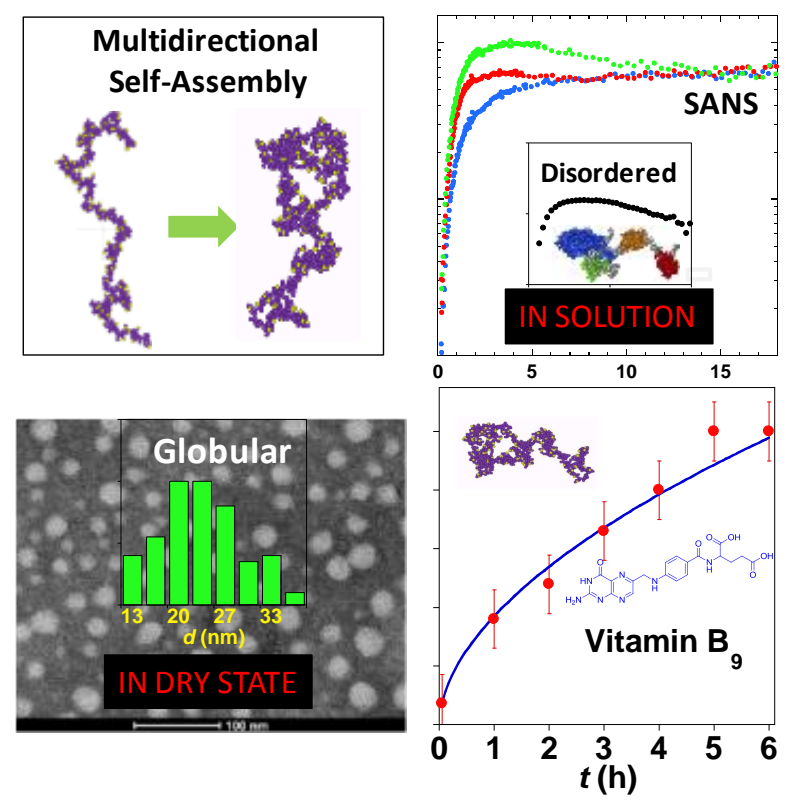